\title{
EXPLORATION IN THE SOLAR SYSTEM WITH ELECTRIC SPACECRAFT
}

\author{
ERNST STUHLINGER \\ NASA George C. Marshall Space Flight Center
}

The landings of instrumented probes and astronauts on the Moon and the short glimpses at Venus and Mars that distinguished the spaceflight program of the last decade yielded such an impressive wealth of new knowledge that the President, in his programmatic speech of 1970, mentioned the continuing exploration of the solar system as one of the national goals during the decade of the seventies.

This exploration will be accomplished with unmanned spacecraft, except for the remaining three Apollo flights in 1971 and 1972 and Skylab in 1973. Planetary exploration will include photographic coverage of the surfaces of the celestial bodies; closeup pictures of specific surface features; magnetic and gravitational measurements; observations of atmospheres, ionospheres, and radiation belts; analysis of surface material in situ; and, as far as possible, the return of surface samples for careful chemical and mineralogical analyses and for age determinations.

In addition to the Moon and the nine planets, two other groups in the solar family recently have aroused great interest among astronomers and cosmologists: asteroids and comets. Asteroids, unlike heavier celestial bodies, have not been subjected to heavy bombardment by meteoroids since the time of their formation about 4.5 billion $\mathrm{yr}$ ago. They are expected to consist of undisturbed and unmodified primordial planetary matter not to be found at any other place in the solar system. Comets are of particular interest because they are covered, in all likelihood, by a thick layer of frozen material, such as water, ammonia, methane, cyanogen, perhaps even formaldehyde and other more complex compounds, which the nucleus of the comet accumulates while slowly moving through its apogee far away from the Sun, in many cases even beyond the orbit of Pluto. As the comet moves through its perigee, the Sun gradually melts and removes the frozen cover. A probe cruising through the cometary tail would be able to sample and analyze the interplanetary matter that a comet collects in space and displays in the proximity of the Sun.

Missions to planets, asteroids, and comets, as outlined in this exploration program, have several features in common. Each mission will last between 1 and several $\mathrm{yr}$; each spacecraft must possess a considerable capability to 
maneuver and to change its flight velocity during the entire duration of the mission; the payload capability should be large; and the amount of available electric power for the transmission of data should be high. Some of these features of planetary missions are listed in table $I$, in comparison with less demanding missions to other targets. It is obvious that the requirements of planetary missions exceed those of other missions by a considerable margin. The question is well justified, therefore, whether a propulsion system other than the conventional chemical rocket motor may be appropriate for the long and demanding transfer from the vicinity of Earth to a planet, an asteroid, or a comet. The answer to this question should be an emphatic yes. Electric rocket motors, more specifically ion motors, seem to be ideally suited for space missions that extend over time periods of 1 or more yr. In fact, the electric propulsion system, when used on planetary missions, offers a high incremental velocity, an almost unlimited reignition capability, a large payload fraction, a long operating lifetime, and a sizable electric power source available for data transmission after the target has been reached. Electric-propulsion systems have been under study and development for many years, and they are now ready for applications.

An electric-propulsion system requires a source of electric power on board the spacecraft. Two prime sources of electric energy are appropriate for electric spacecraft propulsion, solar electric power supplies, and nuclear electric power plants. Solar electric power supplies have been used in space with great success for over $12 \mathrm{yr}$. They have an operable lifetime of years, and their present conversion efficiency of about 12 percent is entirely satisfactory for flight missions as envisioned during this decade. Solar electric power holds great promise for electrically propelled spacecraft that orbit around or land on Mercury, Venus, or Mars; meet with a comet; or descend to the surface of an asteroid. Even sample-return missions to all these targets will be possible. The decrease of solar energy flux with increasing solar distance makes solar electric power sources less capable near the outer planets. Highly elliptic orbiters around Jupiter, and flybys near the more distant planets, would still show considerable payload gains if an electric stage were used on the spacecraft; however, in view of the large amount of electric power needed to transmit observational data from these remote targets, nuclear electric power sources will be preferable on missions to the outer planets. Also, nuclear electric power

TABLE 1.-Classes of Rocket Flight Missions

\begin{tabular}{l|l|r|r}
\hline \multicolumn{1}{c|}{ Mission } & \multicolumn{1}{|c|}{ Timespan } & $\begin{array}{c}\text { Flight distance, } \\
\mathrm{km}\end{array}$ & $\begin{array}{c}\text { Total velocity increment, } \\
\mathrm{km}-\mathrm{s}^{-1}\end{array}$ \\
\hline Ballistic & Minutes & $10^{3}$ & 5 \\
Orbital & Hours & $10^{4}$ & 10 \\
Lunar & Days & $10^{6}$ & 25 \\
Planetary & Years & $10^{9}$ & 50 \\
\hline
\end{tabular}


will enable a spacecraft to achieve a circular orbit even around the remotest planet. At present, nuclear electric power sources for planetary flight in the kilowatt range are not yet available. The first power plants of that kind may be ready for use toward the end of this decade.

Several different kinds of electric propulsion systems have been under study, among them the resistojet, the arc jet, the ion engine, and the plasma engine. For planetary missions, the ion engine is the best choice; the following description will concentrate on this type of electric propulsion. However, the basic relations to be described apply to all systems that need an electric power source.

In an ion rocket, the exhaust beam consists of ions; they are accelerated within the thrustor by an electrostatic field. Before entering the accelerating field, the propellant atoms must be ionized. The exhaust velocity of the ions $v$ is a function of their specific charge $\epsilon / \mu$ and the potential difference across the accelerating field $U$ :

$$
v=\sqrt{\frac{2 \epsilon U}{\mu}}
$$

The beam of ions represents a current $I$ according to

$$
I=\frac{\dot{M} \epsilon}{\mu}
$$

where $\dot{M}$ is the propellant consumption. The product

$$
U I=W
$$

represents the power contained in the beam. The thrust force $F$ exercised by this ion beam upon the thrustor is expressed by the relations

$$
F=\dot{M} v=\frac{2 W}{v}=W \sqrt{\frac{2 \mu}{U \epsilon}}
$$

An electric rocket has three main components: payload, propellant, and power source. The mass of the thrustor, which is small compared to the mass of the power source, is usually included in the mass of the power source to simplify computations. The power source is characterized by its "specific mass" $\alpha$, measured in kilograms per watt. The lower the specific mass, the more attractive the power source. On the basis of existing technologies, a solar electric space power source can be built with a specific mass on the order of 0.03 to $0.02 \mathrm{~kg} / \mathrm{W}$. An improvement to 0.015 or even $0.01 \mathrm{~kg} / \mathrm{W}$ may be expected toward the end of the decade. 
The performance of a chemical rocket under no-drag and no-gravity conditions is expressed by the well-known Tsiolkovskiy equation (explained in fig. 1(a)):

$$
\frac{M_{L}}{M_{0}}=e^{-u / v}
$$

It says in essence that the payload capability $M_{L} / M_{0}$ or the terminal velocity $u$ of a rocket increases continuously with increasing exhaust velocity ${ }^{1} v$ as shown in figure 2(a). The corresponding equation for electric rockets is shown in figure $1(b)$. This equation contains the variables $\alpha$ (specific mass, measured in kilograms per watt) and $T$ (total propulsion time). For chemical rockets, $\alpha=0$;
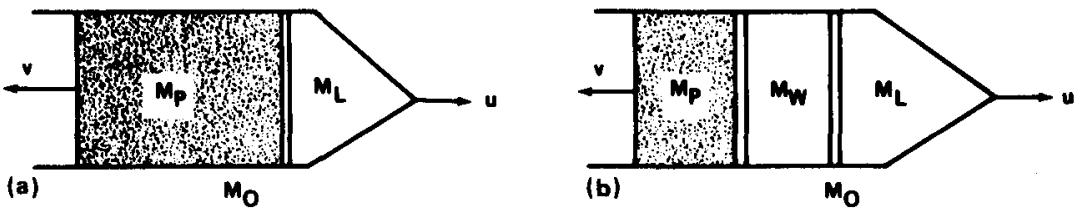

Figure 1.-Equations for two rocket systems. (a) Chemical (endogenous). (b) Electric. (exogenous).

Figure 2.-Payload ratio as a function of exhaust velocity and terminal velocity. (a) Chemical or endogenous systems. (b) Electric or exogenous systems.
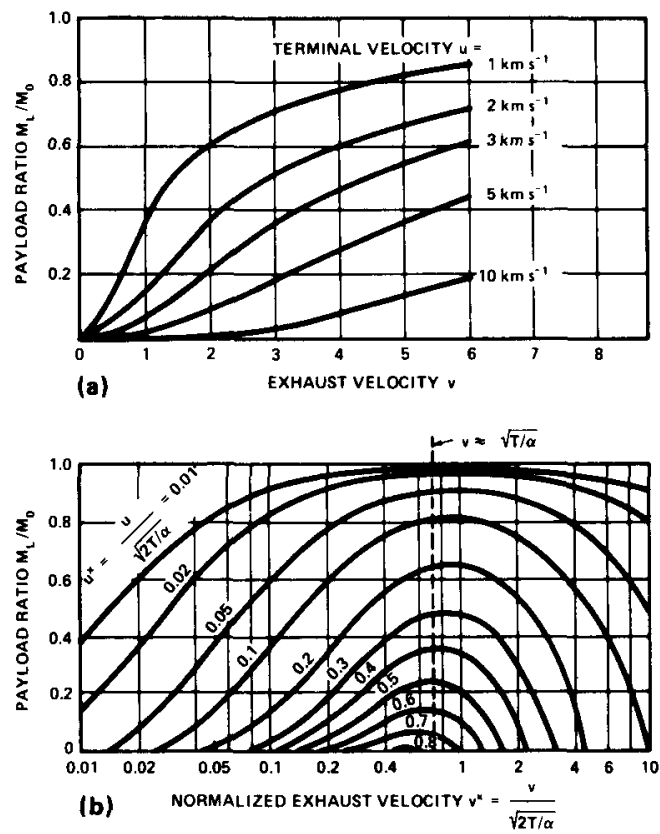

${ }^{1}$ Rocket engineers frequently use the term "specific impulse" $I_{\mathrm{sp}}$ instead of exhaust velocity $v$. Generally $I_{\mathrm{sp}}=v / g_{0} ; g_{0}=$ Earth's gravitational acceleration. 
in this case, the equation for electric rockets reduces to equation (2). Figure $2(b)$ indicates that an electric rocket, for a given terminal velocity $u$, a given specific mass $\alpha$, and a given total propulsion time $T$, has an optimum exhaust velocity $v$ at which its payload ratio $M_{L} / M_{0}$ is a maximum. The designer will choose this optimum exhaust velocity to obtain a maximum payload ratio. The physical reason for the existence of an optimum exhaust velocity is obvious. At higher exhaust velocities, the increase in power supply mass would reduce the payload, and at lower velocities, the necessary increase in propellant mass again would reduce the payload. The following approximations can be obtained from the electric rocket equation and other well-known relations:

$$
\begin{gathered}
v_{\mathrm{opt}} \approx \sqrt{\frac{T}{\alpha}} \\
a_{\max }=\frac{F}{T} \approx \sqrt{\frac{1}{\alpha T}}
\end{gathered}
$$

where $a_{\max }$ is the maximum acceleration obtainable with negligible payload. The simple relations expressed by equations (1) to (4) permit a quick assessment of the design requirements and performance capabilities of an electric propulsion system. However, for careful optimization studies and trajectory computations, an analytical method (Irving and Blum, 1959) has found wide application. Combining equations (1) and (4), we obtain

$$
\begin{gathered}
a_{t}=\frac{F_{t}}{M_{t}}=\frac{\dot{M}_{t} v_{t}}{M_{t}} \\
W=\frac{\dot{M}_{t} v_{t}^{2}}{2}
\end{gathered}
$$

hence

$$
-\frac{d M}{M_{t}^{2}}=\frac{a_{t}^{2}}{2 W} d t
$$

and

$$
\frac{1}{M_{T}}-\frac{1}{M_{0}}=\frac{1}{2 W} \int_{T} a_{t}^{2} d t
$$

The terminal mass $M_{T}$ consists of power source and payload. Obviously, a maximum payload will be obtained when the integral on the right-hand side is a minimum. It is the task of the project planner to find that trajectory which, 
under the constraints of starting point, target point, total traveltime, total initial mass, total available power, and specific mass of the power source, makes the integral a minimum. This problem can be solved with the methods of variational calculus. In fact, computer programs exist already for numerous planetary trajectories and their optimization, and specific missions (for example, a rendezvous with asteroid Eros in 1975 or 1977) can be programed and computed easily. The computation results in optimum figures for the exhaust velocity, the program for thrusting and coasting periods, the guidance program, the actual payload capability, etc.

It is obvious from equations (1) and (4) that the thrust acceleration of an electrically propelled spacecraft will always be low, on the order of $10^{-4} g_{0}$. However, the thrust force on planetary missions will always act over a long period of time, on the order of months or even years. For this reason, the total impulse generated by an electric-propulsion system is of considerable magnitude. In fact, an ion thrustor powered with $16 \mathrm{~kW}$ of electric power and operating for 350 days generates the same total impulse as the hydrogenoxygen rocket engine RL 10 used on the second stage of the Saturn I rocket (six engines) and on the Centaur rocket stage (two engines). (See table II.)

Before the propellant particles can be subjected to the accelerating force of an electric field, they must be ionized. Three different ionization methods have been developed to a high degree of efficiency and reliability: the electron bombardment method (Kaufman engine), developed at the NASA Lewis Research Center; the radiofrequency ion source (Loeb engine), developed at the University of Giessen in West Germany; and the contact ionization method. developed mainly at Electro-Optical Systems Corp. and at Hughes Research Laboratory. All three systems fulfill the requirements of an electric propulsion system, and all have undergone long-time laboratory testing. Furthest advanced in development, testing, and flight applications is the Kaufman engine in which ionization of the vaporized propellant is accomplished by electron bombard. ment; it is shown in figures 3 and 4.

The first application of an electric propulsion system to a space probe, as far as publicly known, occurred in 1964 on the Soviet spacecraft Zond 2. Numerous electric thrustors for attitude and station control were used on U.S. satellites, as shown in table III. An ion thrustor for prime propulsion was applied on the U.S.S.R. space probe Yantar in 1969. Two American test vehicles for ion thrustors, SERT 1 and 2, were launched in 1964 and 1970. Although not completely successiul, they definitely proved the proper functioning of ion propulsion systems under space conditions, and they established full confidence in this method of rocket propulsion. The SERT 2 test vehicle with two $1 \mathrm{~kW}$ ion engines is shown in figure 5. Design lifetime of SERT 2 was $1 \mathrm{yr}$. Project cost, including the Atlas/Agena carrier vehicle, amounted to approximately 12 million dollars.

Experience shows that a thrustor designed for about 2.5 to $3 \mathrm{~kW}$ power consumption represents an optimum thrustor size. Thrustors of this type can 


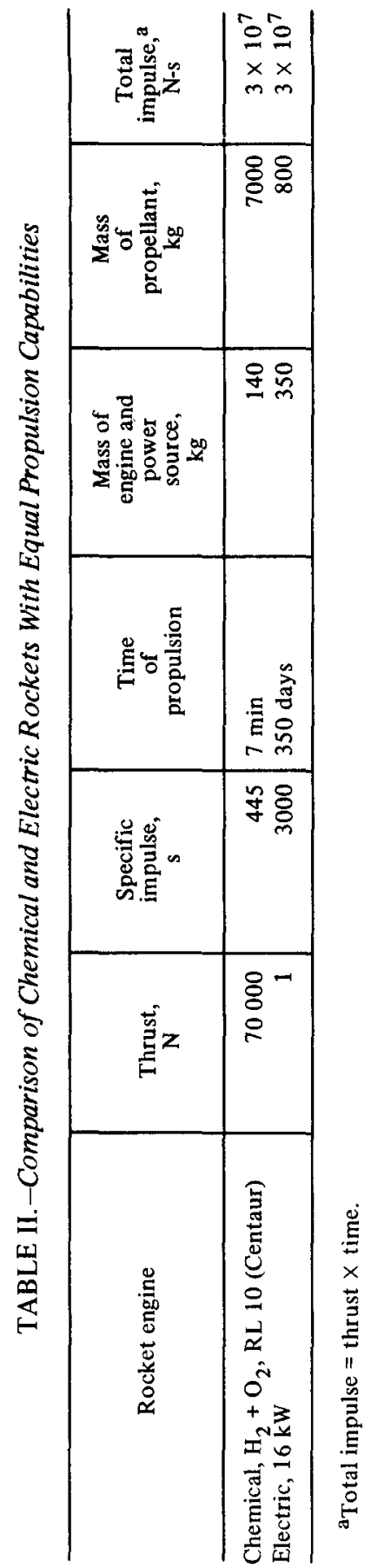




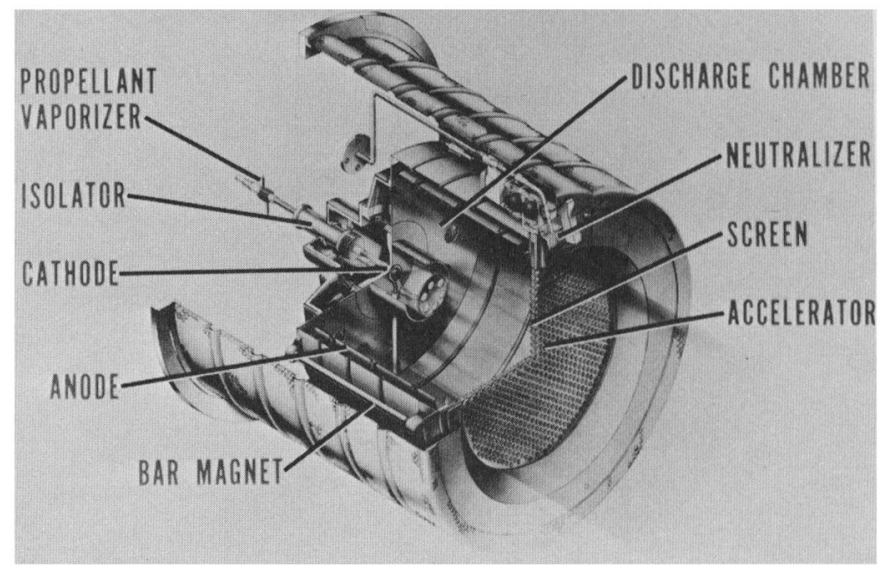

Figure 3.-Cut-away drawing of ion engine (electron bombardment or Kaufman type).

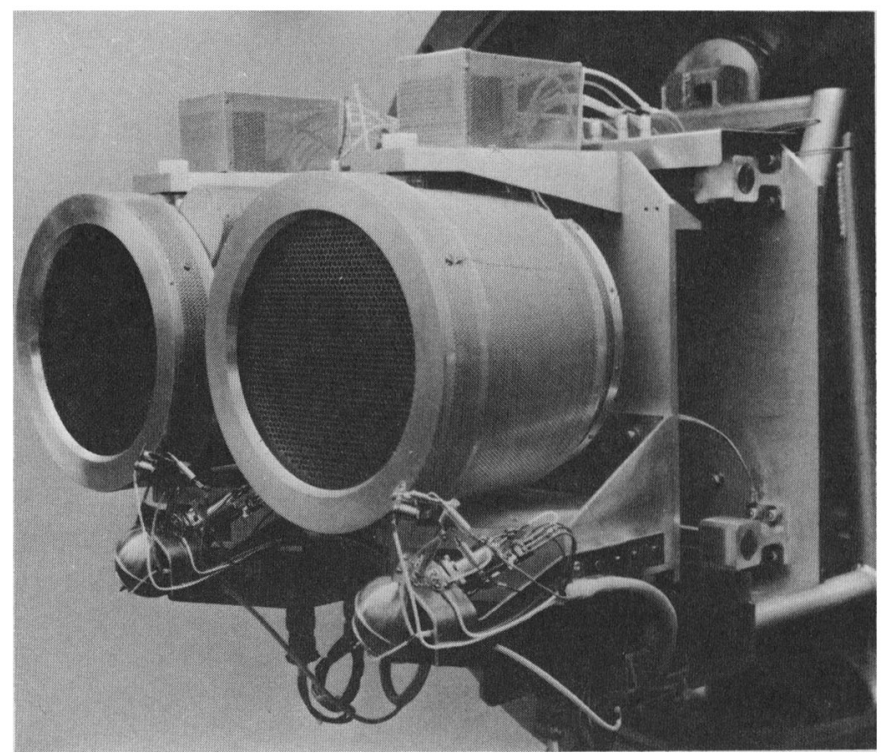

Figure 4.-Cluster of two ion engines (electron bombardment or Kaufman type).

be clustered easily for higher power and thrust levels. A propulsion system with three thrustors, consuming $8 \mathrm{~kW}$ of power, would be an adequate system for "easy" planetary missions. More demanding missions could be carried out with vehicles consisting of two or three modules of the $8 \mathrm{~kW}$ type. An artist's conception of an electrically propelled spacecraft is depicted in figure 6 . 


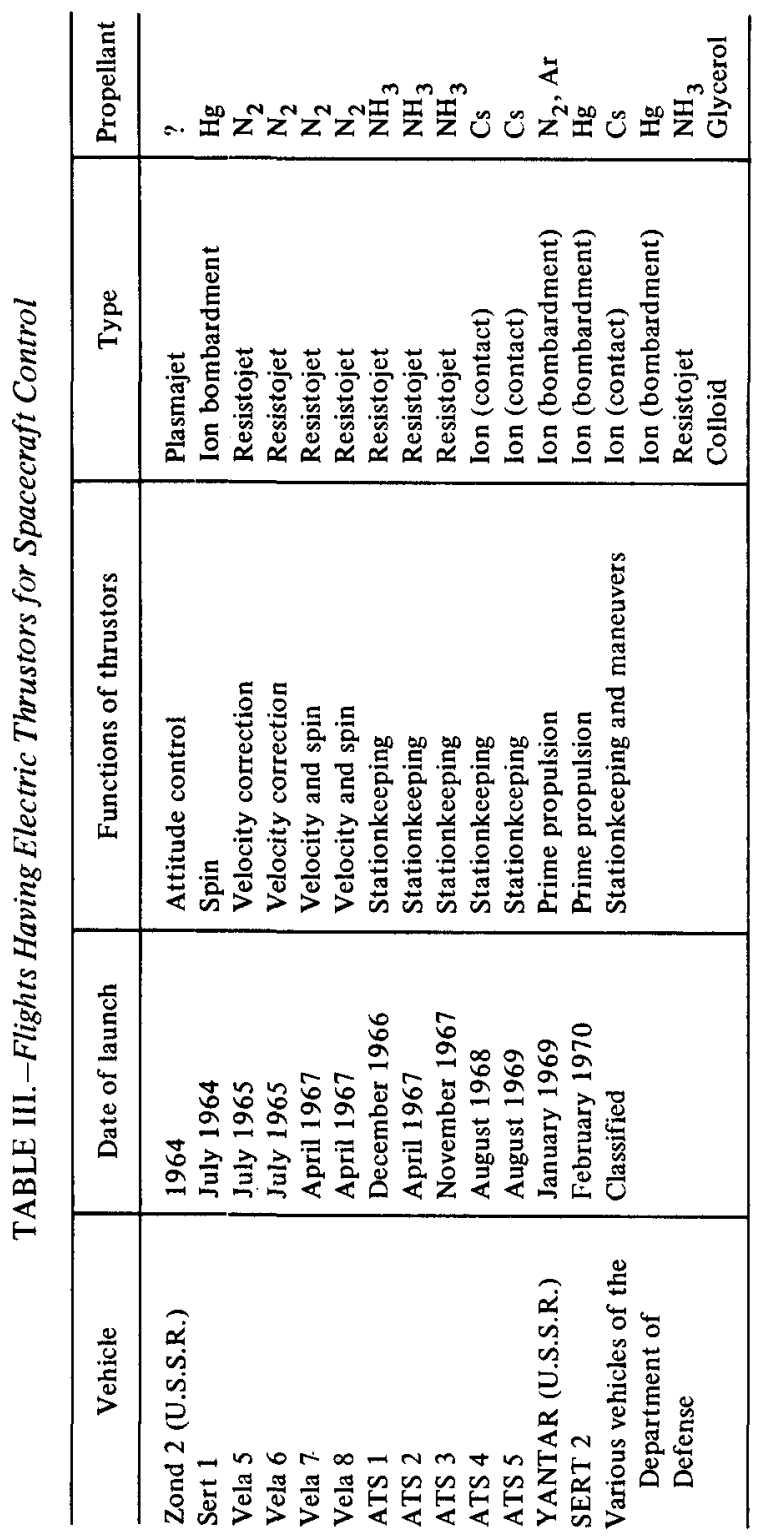




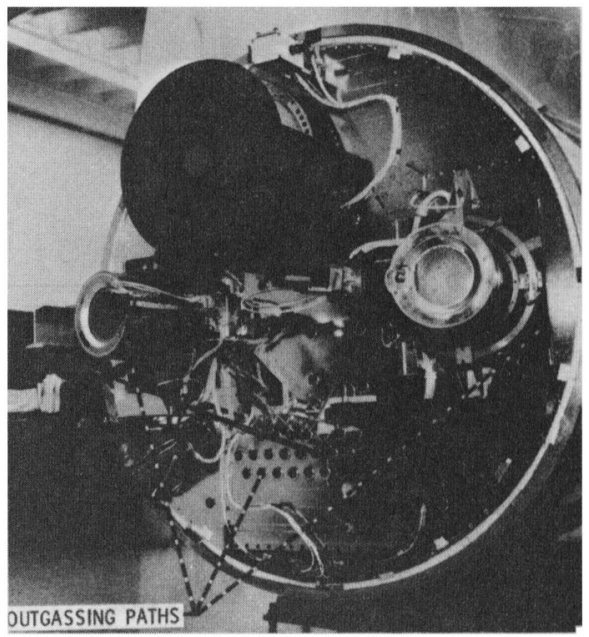

Figure 5.- Rear view of SERT 2 with two electric thrustors.

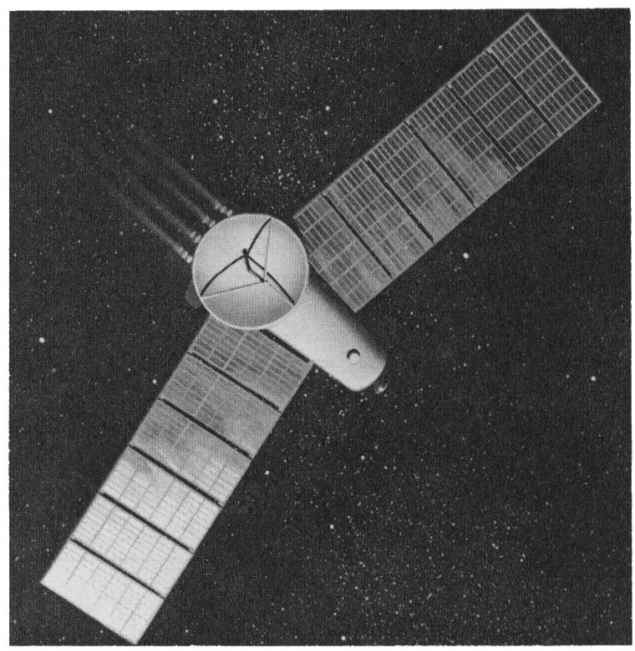

Figure 6.-Solar electric spacecraft on flight to Mars.

Table IV lists a number of desirable flight missions throughout the solar system that could be accomplished with electrically propelled vehicles. The first part contains missions powered with solar electric power; three different vehicle sizes and power levels are envisioned. The second part, containing more demanding missions to the outer planets, is based on a future nuclear electric power source of about $150 \mathrm{~kW}$. 
TABLE IV.-Potential Missions for Electrically Propelled Spacecraft

\begin{tabular}{l|r|r|l|r}
\hline Power source & Departure & Arrival & Destination & $\begin{array}{c}\text { Electric power, } \\
\text { kW }\end{array}$ \\
\hline Solar electric & 1974 & 1975 & Asteroid flyby (Eros) & 8 \\
& 1976 & 1977 & Asteroid landing (Eros) & 8 \\
& 1976 & 1976 & Venus orbiter & 16 \\
& 1976 & 1978 & Comet (Kopf) & 24 \\
& 1977 & 1978 & Execliptic & 8 \\
& 1977 & 1978 & Solar probe (0.1 AU) & 16 \\
& 1977 & 1979 & Jupiter flyby (or highly & 24 \\
& & & elliptic orbiter) & \\
& 1977 & 1980 & Saturn flyby & 24 \\
& 1978 & 1978 & Mercury orbiter & 16 \\
& 1978 & 1978 & Mars orbiter & 24 \\
& 1978 & 1979 & Asteroid landing & 24 \\
& 1978 & 1980 & Comet (Encke) & 24 \\
& 1979 & 1980 & Mars landing & 24 \\
& 1979 & 1982 & Uranus flyby & 24 \\
& 1980 & 1982 & Jupiter orbiter & 150 \\
& 1980 & 1982 & Comet (d)Arrest) & 150 \\
& 1981 & 1984 & Saturn orbiter & 150 \\
& 1981 & 1985 & Neptune orbiter & 150 \\
& 1982 & 1986 & Comet (Halley) & 150 \\
& 1982 & 1986 & Uranus orbiter & 150 \\
& 1982 & 1987 & Pluto orbiter & 150 \\
& & & \\
& & &
\end{tabular}

NOTE.-All launch vehicles would be of the Titan class except Eros flyby, which would use the Atlas/Centaur; see p. xxy.

The total masses of the spacecraft listed in table IV, at the beginning of their planetary trajectories, vary between about 500 and $6000 \mathrm{~kg}$. Most of them could be launched also with a space shuttle instead of a Titan. In this case, a chemical kick stage must be provided to accelerate the spacecraft from the shuttle orbit into the planetary transfer trajectory.

The list of potential projects on table IV starts with an asteroid flyby. Asteroid Eros has a fairly eccentric trajectory; every $2 \mathrm{yr}$ it approaches the Sun with a perigee of only 1.3 or $1.2 \mathrm{AU}$. In 1977, the Earth-Eros distance will be less than one-third the shortest Earth-Mars distance.

The proposed asteroid flyby mission in 1975 would represent a relatively easy mission with modest velocity requirements and with a guidance system that would have to guide the spacecraft only to a distance of about $100 \mathrm{~km}$ from the asteroid for photography, temperature measurements, and some other unsophisticated observations. Most of the instruments for this flyby mission would be available from previous projects, such as Surveyor, Mariner, and Lunar Orbiter. The spacecraft could be launched with an Atlas/Centaur. Although this flight would not yet provide all the desired information on Eros, it would obtain important data on size, shape, mass, rotation, surface features, 
and other properties of the asteroid that must be known for a successful follow-on project, the landing and data-return mission to Eros. Equally important would be another objective met by this simple flyby mission, a full-scale test flight of the complete electric propulsion system. From the standpoint of the spacecraft engineer, such a test flight would be very desirable before a spacecraft as complex and expensive as an asteroid landing and sample-return vehicle is committed for flight. Evidently, a project of this kind would not only be a highly valuable preparatory step for an asteroid lander mission but also a most important achievement with respect to the evolution of electric spacecraft for planetary exploration. Although data from the flyby mission would become available not more than a year before the launching of the lander mission, these data would be valuable for instrument settings and other details of the lander mission and as a confirmation of design data chosen for the lander. If the flyby should reveal severe deviations of the asteroid features from anticipated properties, the launching of the lander would have to be postponed.

None of the projects listed in table IV has attained the status of an approved project; all of them have only been the subjects of very preliminary studies. However, the list of potential projects may indicate the great promise that electric propulsion holds for a broad program of planetary exploration that could begin in the midseventies and would provide a rich harvest of knowledge of our entire solar system.

\section{REFERENCE}

Irving, I. H., and Blum, E. K. 1959, Comparative Performance of Ballistic and Low Thrust Vehicles for Flight to Mars. Vistas Astronaut. 2, 191.

\section{DISCUSSION}

WETHERILL: I think it would be a mistake to overemphasize differences in the merits of cometary and asteroidal missions. There are considerable savings in a program of missions using similar spacecraft; the cost per launch is much less for multiple missions than for single missions. These savings could be realized by development of a multipurpose solar electric spacecraft suitable for both asteroid and comet missions. From the point of view of scientific priorities, $I$ think as much emphasis should therefore be placed on the sum of the value of these two types of missions as on the difference in their value.

1 understand that the NASA Office of Advanced Research and Technology (OART) is requesting funds for a solar electric interplanetary mission in the budget currently before Congress, and I wonder what plans exist for obtaining scientific advice in planning these missions.

SOBERMAN: In the past, mission definition has taken place with little input from the scientific community. At the time of the "Announcement of Flight Opportunity," the proposing scientists are faced with a vehicle for which at least a preliminary design exists. This "preliminary design" in practice is difficult, if not impossible, to modify. Even in the case of the Grand Tour missions, the so-called scientific definition phase must contend with the constraints of the TOPS vehicle.

I propose that NASA set up a definition team of scientists to work with the mission planners at the earliest stages so that the result would be system-optimized to perform the science. 
DWORNIK: At NASA Headquarters, the Office of Space Science and Applications (OSSA) in fact does conduct an early mission definition phase that involves the scientific community. Scientists were invited to participate in the early planning phase of the Viking and the Mariner-Venus ' 73 and Mariner-Mercury ' 73 missions in order that spacecraft and mission constraints would not freeze out certain types of experiments. Specifically, the Mariner 1973 program actually altered early spacecraft and mission constraints to accommodate experiments.

BARBER: In the final recommendations, OSSA requires sufficient information for mission selection. Also at NASA Headquarters, OART is trying to provide some of this information for evaluation by OSSA. The initial thrust of our studies is to decide the technology feasibility of such small-body missions. We at OART are now at the point where we have a fairly systematic approach to the technology problem. The remainder of the work is to evaluate the scientific impact of the solar electric trajectory and spacecraft. The question as to what should be the science is not being considered at this time because the investigation is primarily technology oriented. When the actual mission is chosen, one would expect to turn to the scientist. At that time, the primary responsibility will transfer from OART to OSSA. 